The chemical effects are dealt with in Part 2 by Dr. M. Lefort, who discusses what has now become known as the radiation chemistry of aqueous solutions.

Part 1, by Dr. L. H. Gray, represents something between text-book material and a monograph. The discussion is based essentially on the earlier concepts of D. E. Lea; there is no reference to the more recent work of Kramers which, from the physical point of view, is rather fundamental for the action of ionizing radiations in liquid systems. In Part 1 , and particularly in Part 3, by Dr. W. M. Dale, there is probably too great a tendency to apply results obtained on very dilute aqueous solutions to biological systems.

It would, perhaps, not have been inappropriate to give rather more prominence to the work of Hollaender and Latarjet on the possible relationship between radiobiology and photobiology. It is clear that, in general, the behaviour of biological systems towards radiation does not depend so much on the energy of the incident primary particle or quantum but rather on the energy-levels of the particular compounds which are involved. Thus, even in the case of highenergy radiations, electronic levels of only a few electron volts may ultimately be of great importance for the chemical and related biologieal effects.

Part 3 deals mainly with the chemical action of ionizing radiations on certain selected organic compounds (for example, thiourea) in aqueous solution. There is little discussion of the actual biochemical effects produced by ionizing radiations, a subject which has become known, to a large extent, through the work of Hevesy and Forssberg; only one very brief reference to Hevesy's work has been given, and this dates back to 1945 .

In spite of certain shortcomings, this volume contains much stimulating information and a useful collection of references, and one is indebted to the

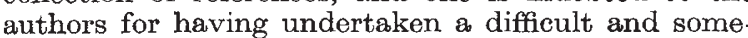
what unrewarding task. The specialist concerned with one aspect of the wide and diverse field of the chemical and biological action of radiations often feels the need for comprehensive accounts of the many other fields of work closely related to his own. This new series, when complete, should go a long way towards fulfilling this need. A special word of praise and encouragement is due to Dr. Haïssinsky, who is to be wished every success in the further development of this collection of monographs.

J. WEISS

\section{HANDBOOK OF REACTOR PHYSICS}

Reactor Handbook : Physics

Prepared by the United States Atomic Energy Commission. (Selected Reference Material on Atomic Energy.) Pp. xvi + 790. (London: MeGrawHill Publishing Company, Ltd., 1955.) $90 s$.

THIS handbook is one volume in a series sponsored by the United States Atomic Energy Commission with the avowed intention of providing a condensed source of reliable data and reference information in the field of reactors. The volume is devoted to reactor physics and radiation shielding, the latter subject occupying approximately a quarter of the book.

The first two chapters are devoted to experimental methods and nuclear physics. The former refers to neutron sources and detectors and, in outline, to methods of measurement of fundamental and integral nuclear and reactor data. The latter is primarily concerned with an extensive compilation of the properties of nuclei in tabular and graphical form.

The remainder of the section on reactor physics is devoted to the kinetic theory of neutrons and reactor statics and dynamics. The kinetic theory of neutrons discusses some of the approximations which are commonly used in the analytical solution of Boltzmann's equation. This treatment is rather sketchy for the mathematician, but the principles are discussed and references to more detailed papers are given. The application of these approximations to steady-state reactor problems is given, but attention is concentrated on homogeneous reactors. No theoretical treatment of heter geneous reacturs is given, but the results of various integral measurements, including experiments to determine critical factors, are prefaced by a statement of simple formulæ for computing the characteristics of an infinite lattice reactor. Many results are given, but it is disappointing that no attempt is made to correlate these results with theoretical estimates made in different ways. The chapter on reactor dynamics contains a useful discussion, with numerical examples, of the effect of changes in reactivity on the neutron intensity. Consideration is also given to the criteria for stability in a power-producing reactor, taking into account the problem of heat dissipation and the power and temperature reactivity coefficient. Typical calculations for control rods complete this chapter.

The section of the book on shielding contains chapters dealing with sources of neutrons and gammarays in a reactor, radiation tolerances, neutron and gamma attenuation and heating including the effect of geometry and ducts, optimum dimensions of shields and a tabulation of general properties of shielding materials. As in the previous section, the tabulation of data is extensive and in general very useful. The number of experimental results from shielding experiments which are quoted is rather small, and little attempt is made to recommend procedures.

The authors of the handbook are clearly aware of the limitations imposed on the contents and on its presentation by the rigid target date for the production of this edition. As a consequence, however, some of the information given is difficult to digest, and the reader must be experienced in the field and prepared to work hard in order to extract the information leally required from such a handbook. An outstanding exception to this is the tabulation of many useful general and nuclear properties of materials used in reactor's. Modifications to any future editions, which would make this handbook of outstanding value both to newcomers and to the experts in this field, are a general unifying introduction, a constructive criticism of theories and experiments and their limitations, and, in particular, a recommendation of specific methods and data which correlate with experiment for specific problems.

The production of this handbook was obviously a formidable task, and the authors are to be congratulated on producing a volume of this kind in the time available. Nevertheless, as the members of the review board have indicated in their preface, "the ultimate success of the handbook will depend a great deal upon the refinements in subject-matter and presentation which will come about in subsequent revisions".
Peter W. Mummery 